\title{
49
}

\section{Cyclomatic complexity as a measure of the structure of knowledge-based/expert systems}

\section{T. T. Moores}

Department of Information Systems, City Polytechnic of Hong Kong, 83 Tat Chee Avenue, Kowloon, HONG KONG.

\begin{abstract}
The "structure" of a program is often used as an indicator of the likely error-proneness of a system (and thus its quality). This paper reviews the applicability of cyclomatic complexity as a measure of the structure of knowledge-based systems (KBSs). Threshold values are sought above which $80 \%$ of error-prone programs are captured. The results obtained suggest that cyclomatic complexity substantially outperforms lines of code as a measure of the error-proneness of KBSs.
\end{abstract}

Keyword Codes: D.2.2; D.2.5; D.2.8

Keywords: Tools and Techniques; Testing and Debugging; Metrics

\section{INTRODUCTION}

Measures of complexity is seen as the best indicator of error-proneness, or the likelihood of a piece of software to have an operational fault (e.g., [1,2]). "Good structure" has often been described as the ability of a system to resist errors even when changes are made to some part of its sub-structure [3,4]. It is on this basis that controlling the structure of a program has a direct bearing on the quality of the overall system. The classic studies for conventional systems are McCabe[5] and Henry \& Kafura $[6,7]$. Clearly, "quality" is not simply an issue of the number of bugs, but it can often be reduced to such a judgement. The question to be addressed in this paper is whether similar metrics can be derived for KBS languages such as Prolog. For the sake of simplicity, the terms "knowledge-based system" and "expert system" will be considered to be interchangeable and the term KBS will be used throughout this paper.

Very few metrics have been developed for knowledge-based systems (KBSs). Those KBS metrics which do exist have not been extensively validated and are mainly suggestions of the types of measurements to make [8-10]. Attempts to define 'structure' have included: counting the different types of node in a Rete-network [11], modelling the way data is partitioned within a Prolog clause [12], or modelling the location of data within a clause and then measuring the structure of the resultant model [13]. Outside these references, however, there is little or no work being done on metrics for KBSs.

This paper will test the assumption that structure metrics can be developed for Prolog-based systems without needing to specifically address the "KBS-ness" of the system. This simplification would lead to the useful conclusion that characteristics of a development environment becomes an inherent part of any metrics model and there is no need for any special (KBS) definition of the term "structure". On the grounds of its relative popularity, the attempt here will be to apply cyclomatic complexity [5] to Prolog, with lines of code (LOC) taken as a baseline metric. It will be expected that LOC is not a good structure metric. Section 2 will describe the two main variants of the cyclomatic complexity metric $(v(G)=e-n+2$ and $v(G)=$ No. of conditionals +1$)$. Section 3 will present the results of applying the 
cyclomatic complexity metric to 80 commercially-developed LPA Prolog programs. For a description of the Prolog language, the interested reader is refered to [14-16].

\section{CYCLOMATIC COMPLEXITY}

Cyclomatic complexity [5] is a graph-theoretic structure metric which attempts to define the procedural complexity of a program in terms of flows through a graph. Blocks of code are represented as nodes while decision-points are represented as arcs joining one node to another. By transforming a program into a graph, a measure of program complexity would then be a measure of the number of paths through this graph. Paths are identified by defining a program as a strongly connected graph, G, which has $n$ vertices, $e$ edges and $p$ connected components. The cyclomatic number, $\mathrm{v}(\mathrm{G})$, can then be calculated as:

$v(G)=e-n+2 p$

where, $\mathrm{p}=1$ (for one component). The component count, $p$, signifies the number of separate routines within a program. In most studies, $p$ is taken to be one. The equation for cyclomatic complexity can also be reduced to a count of the number of predicates (such as IFs, WHILEs, etc.), plus one. In practice, McCabe suggests that it is more convenient to count conditionals and so the refined measure of cyclomatic complexity becomes:

$v(G)=$ No. of conditionals +1

Some care needs to be taken over the fact that compound conditionals are actually disguised IFs (e.g., IF "C1 AND C2" THEN.. becomes IF C1 and IF C2 THEN..); while CASE statements are actually multiple IFs, where a CASE statement with $\mathrm{N}$ conditions would have to be re-written as $\mathrm{N}-1$ IF statements. The distinction between well-structured and badly-structured programs is that the program graph of well-structured programs can be reduced sequentially by removing nodes with only one input and one output until there is only one node left.

The idea of transforming a program into a graph of pathways proved to be an appealing and influential concept. The first reactions, however, were to redefine the metric in order to accommodate problems in the way the graph represented certain program structures. New variations of the metric quickly emerged where more emphasis was placed on the bounds of ELSE statements[17], arcs at a node forming "knots"[18], the flow of both control and data[19] and the nesting depth of statements (e.g., [20]). The most important use of these graph-theoretic measures of complexity has been to explain the number and distribution of errors[21,22]. The complexity of a program has also been related to the difficulty of developing the program[23], the use or reachability of particular nodes[24], while later studies generated similar graphs on the basis of the system design or specification (e.g., [25]).

Similar to many structure metrics, however, it is sometimes difficult to detect how the elements of the program are to be mapped onto the appropriate metric elements. By abstracting what a program is to a graph of nodes and arcs it becomes confusing to know whether we are measuring syntactical or psychological program complexity[26]. McCabe clearly wants to measure both syntactical and psychological complexity, but the bridge from its syntactical measurement to its psychological impact is uncertain[27]. For instance, Halstead's model of effort, $E$, has been found to correlate with construction time, the number of errors, and debugging time significantly better than cyclomatic complexity[28]. The lack of support for relating measures of complexity to what programmers see as "complex" and thus being error-prone is a typical criticism of structure metrics (e.g., [29]). Even in view of these criticisms it still appears that cyclomatic complexity remains an appealing (if unproven) structure metric. 


\section{APPLYING STRUCTURE METRICS TO PROLOG}

The aim of the structural analysis here is to deduce a metric threshold value, T, for LOC and v(G) above which Prolog programs tend to be error-prone. A set of 80 commercially-developed LPA Prolog programs were analysed, of which 46 had a total of 115 post-release errors (i.e., $57.5 \%$ of the programs had errors). Such errors record faults found by users and beta-testers after the product had been released. Although structure metrics are more typically related to errors found during testing, no reports detailing coding or testing errors were available. The analysis will focus on detecting an errorprone program regardless of how many errors a particular program might have. The structure metrics being used are:

- lines of code (defined as non-comment, non-blank lines of code);

- cyclomatic complexity, $\mathrm{v}(\mathrm{G})_{1}=\mathrm{e}-\mathrm{n}+2$; and,

- cyclomatic complexity, $\mathrm{v}(\mathrm{G})_{2}=$ No. of conditionals +1 .

The two variants are assessed together because it is not clear at this point which version is likely to work best. The theoretical version, $v(G)_{1}$, requires a definition of Prolog decision points (edges) and blocks of code (nodes). The practical version, $v(G)_{2}$, requires a count of Prolog conditionals only. Cyclomatic complexity is defined here for Prolog in terms of the calls from one predicate to another and has the following properties:

- a single node, $n$, replaces all instantiations of the predicate (such that, a call by any predicate with the same name is represented as a call from a single node with that name);

- a predicate in the body of a clause is represented as an arc, $e$, from the clause head node to the clause body node (where one arc represents all calls between predicates of the same name);

- the number of conditionals contained within a program is equated to the number of unique predicate names, $P$;

- The calculation of cyclomatic complexity is defined as either:

$$
\begin{aligned}
v(G)_{1} & =e-n+2 \\
\text { or, } & v(G)_{2}=P+1
\end{aligned}
$$

Using these definitions a program can be represented as a directed flow-graph from which a measure of cyclomatic complexity can be taken.

Typically, the justification for a good structure metric is one which has a reasonably high (ranked) correlation between the metric value and programs with errors. However, this is of little value to the developers who are more interested in pinpointing exactly those programs within the system which are likely to have errors. Rather than using correlation, therefore, the analysis here will aim to identify a metric threshold value, $\mathrm{T}$, which identifies most (say, 80\%) of those programs with errors. This would allow more directed testing to be carried out and, hence, increase the overall quality of the delivered system with the minimum of effort. For arbitrary reasons, the threshold chosen is the point at which those programs above the threshold represent at least $80 \%$ of programs with one or more recorded errors. Since there are 46 programs with errors, this means looking for the point at which 37 or more of the error-prone programs are above $T$.

Along with this condition, however, it also seems necessary to maximise the proportion of programs which exceed $T$ and have recorded errors $\left(\right.$ Prop $\left._{R}=\max \right)$ and minimise the proportion of programs which have recorded errors but do not exceed $\mathrm{T}\left(\mathrm{Prop}_{\mathrm{W}}=\mathrm{min}\right)$. These two conditions show the success of the metric in terms of its 'strike rate' of identifying error-prone programs (Prop ${ }_{R}$ ), and its fallibility in terms of its failure to flag programs which do, in fact, have errors (Prop $\left.{ }_{W}\right)$. These conditions are therefore defined as:

$\mathrm{S}_{\mathrm{T}}(\mathrm{T})=$ the proportion of programs with errors that exceed $\mathrm{T}$

$=E / N_{E}=E / 46$

Prop $_{\mathrm{R}}=$ the proportion of programs which exceed $\mathrm{T}$ that have errors

$$
=\mathrm{E} / \mathrm{N}_{\mathrm{T}}
$$


Prop $_{\mathrm{W}}=$ the proportion of programs which do not exceed $\mathrm{T}$ but have errors

$=\left(N_{E}-E\right) /\left(N_{\text {total }}-N_{T}\right)=46-E / 80-N_{T}$

where,

$\mathrm{E}=$ number of programs above $\mathrm{T}$ which have errors $(\max =46)$

$\mathrm{N}_{\mathrm{E}} \quad=$ number of programs with errors (total $=46$ )

$\mathrm{N}_{\mathrm{T}} \quad=$ number of programs above $\mathrm{T}(\max =80)$

$\mathrm{N}_{\text {total }}=$ number of programs (total $=80$ )

The value of $S_{\mathrm{T}}(\mathrm{T})$ will vary between 0.00 and 1.00 . In comparing the performance of each of the three structure metrics, therefore. the threshold for each is taken be the highest value of $T$ for which the following are true:

$S_{T}(T) \geq 0.80$ (and where Prop $_{R}=\max$ and Prop $_{W}=\min$ )

Table 1.

Results of metrics at $\mathrm{S}_{\mathrm{T}}(\mathrm{T})=0.80$

\begin{tabular}{lcccc}
\hline Metric & $\begin{array}{c}\text { Highest value of T for } \\
\text { which } S_{T}(T)=0.80\end{array}$ & $N_{T}$ & Prop $_{R}$ & Prop \\
\hline LOC & 74 & 61 & 0.607 & 0.474 \\
$\mathrm{v}(\mathrm{G})_{1}$ & 33 & 58 & 0.638 & 0.409 \\
$\mathrm{v}(\mathrm{G})_{2}$ & 34 & 53 & 0.698 & 0.333 \\
\hline
\end{tabular}

The thresholds for each structure metric are calculated as the highest value of $T$ for which $\mathrm{S}_{\mathrm{T}}(\mathrm{T})=0.80$ (see Table 1). As can be seen, the best metric appears to be the practical version of cyclomatic complexity, $v(G)_{2}$, on the grounds that it:

- requires the fewest number of programs to be tested in order to capture $80 \%$ of the programs with errors (53, as opposed to 58 or 61$)$;

- has the highest Prop $\mathrm{P}_{\mathrm{R}}$ and lowest Prop $\mathrm{W}$ at the required $80 \%$ cut-off point $(0.698$ and 0.333 , respectively).

In other words, by using the threshold $v(G)_{2}>34$, the developers can reduce the number of programs tested by $34 \%$, and still capture $80 \%$ of error-prone programs. The actual threshold chosen for $\mathrm{v}(\mathrm{G})_{2}$, however, would depend on the limits placed on the number of error-prone programs which are allowed to be released, and the number of programs which can be reasonably tested during the final development phase.

It should be noted that six of the largest programs exceed the threshold for all three structure metrics and yet have no documented post-release errors. This appears to be because all six programs are core to the functionality of the system and so were extensively tested before release. Hence, it could be argued that the metrics defined here have only captured programs which have been inadequately tested rather than which have overly-complex structures that make them error-prone. On the other hand, it could also be argued that since fixing a "bug" during testing is cheaper than post-release "fixes" (e.g., [30]), the errors detected within the sample of 80 programs by $v(G)_{2}$ are the sort of expensive repairs which the standard testing procedure has clearly not captured.

\section{SUMMARY OF RESULTS}

The results of the analysis carried out in the previous sections have shown that knowledge-based systems developed using Prolog are amenable to metrics analysis using measurements previously designed for conventional languages. In particular, models of structure have been deduced from the 
sample of 80 LPA Prolog programs, where a program is likely to contain at least one documented postrelease error when,

$v(G)_{2}>34$

where,

$v(G)_{2}=$ No.of unique predicate names +1

Although the values of $v(G)_{1}$ and $v(G)_{2}$ are quite similar (33 and 34 , respectively), $v(G)_{2}$ is clearly the better structure metric because it has a better Prop ${ }_{R}$ 'strike rate', and misclassifies the fewest proportion of error-prone program as being defect-free. The use of $\mathrm{v}(\mathrm{G})_{2}$ is also important since it clearly out-performs LOC and is based on a feature of the Prolog language which is important to the developers: The number of different predicate names that need to be defined in order to implement the system. It is interesting to note that the number of unique predicate names used within a program has also been found to be the dominant variable in models which estimate the size of Prolog programs (e.g., see [31]).

Calculating v(G) early - for instance at the design stage - is a problem which has been investigated recently for conventional systems' development and shows some promise (e.g., [32]). Whether similar early quality assessments can be made for KBSs is a matter of further investigation.

The importance of this study is the clear result that metrics can be applied to KBS development. In its most naïve form, metrics are simply used to assign a value to attributes of system development which can help to answer "How much?" and "How long?" questions. With answers to these questions, the risks associated with KBS development are reduced because the cost and duration of the project can be better estimated. If the results of this study can be repeated, then the conclusion must be that KBS development no longer becomes a risky venture and can be measured and controlled as well as any other form of systems development.

\section{REFERENCES}

1. SCHNEIDEWIND, N.: 'Software metrics for aiding program development and debugging', Proc. AFIPS National Computer Conference, New York, June 1979, pp989-994.

2. DUNSMORE, $\mathbf{H}$. and GANNON, $\mathbf{J}$.: 'An analysis of the effects of programming factors on programming effort', Journal of Systems and Software, 1980, Vol.1, (2), pp. 144-153.

3. SOONG, N.: 'A program stability measure', Proc. AFIPS National Computer Conference, 1977, ppl63-173.

4. YAU, S, and COLLOFELlO, J.: 'Some stability measures for software maintenance', IEEE Transactions on Software Engineering, 1980, Vol.6, (6), pp.545-552.

5. McCABE, T.: 'A complexity measure', IEEE Transactions on Software Engineering, 1976, Vol.2, (4), pp.308-320.

6. HENRY, S. and KAFURA, D.: 'Software structure metrics based on information flow', IEEE Transactions on Software Engineering, 1981, Vol.7, (5), pp.510-518.

7. HENRY, S. and KAFURA, D.: 'The evaluation of software systems' structure using quantitative software metrics', Software - Practice and Experience, 1984, Vol.14, (6), pp.561-571.

8. KAISLER, S.: 'Expert system metrics', Proc. International Conference on Neural Networks, Atlanta, October 1986, pp.114-120.

9. READDIE, M., STRENG, K.-H. and WERMSER, D.: 'KADS metrication', ESPRIT Project 1098, Report SD-G10-001. Produced by SD(Europe) Ltd. \& NTE NeuTech Entwicklungsgesellschaft + Co. KG, 1989. 
10. BEHRENDT, W., LAMBERT, S., RINGLAND, G., HUGHES, P. and POULTER, K.: 'GATEWAY: Metrics for knowledge based systems', Proc. First World Congress on Expert Systems, Miami, December 1991, pp.1056-1067.

11. GUPTA, A. and FORGY, C.: 'Static and run-time characteristics of OPS5 production systems', Journal of Parallel and Distributed Computing, 1989, Vol.7, pp.64-95.

12. MARKUSZ, Z. and KAPOSI, A.: 'Complexity control in logic-based programming', The Computer Journal, 1985, Vol.28, (5), pp.487-495.

13. MYERS, M. and KAPOSI, A.: 'Modelling and measurement of Prolog data', Software Engineering Journal, 1991, Vol.6, (4), pp.413-434.

14. CLOCKSHIN, F. and MELLISH, C.: 'Programming in Prolog', (Springer-Verlag, 1981).

15. BRATKO, I.: 'Prolog Programming for Artificial Intelligence', (Addison-Wesley, 1986).

16. O'KEEFE, R.: 'The Craft of Prolog', (The MIT Press, 1990).

17. MYERS, G.: 'An extension to the cyclomatic measure of program complexity', ACM SIGPLAN Notices, 1977, Vol.12, (10), pp.61-64.

18. WOODWARD, M., HENNELL, M. and HEDLEY, D.: 'A measure of control flow complexity in program test', IEEE Transactions on Software Engineering, 1979, Vol.5, (1), pp.45-50.

19. OVIEDO, E.: 'Control flow, data flow and program complexity', Proc. IEEE Computer Software and Applications Conference, November 1980, pp 146-152.

20. HARRISON, W. and MAGEL, K.: 'A complexity measure based on nesting level', ACM SIGPLAN Notices, 1981, Vol.16, (3), pp.63-74.

21. KITCHENHAM, B.: 'Measures of programming complexity', ICL Technical Journal, 1981, May, pp.298-316.

22. BASILI, V. and PERRICONE, B.: 'Software errors and complexity: An empirical investigation', Communications of the ACM, 1984, Vol.27, (1), pp.42-52.

23. BASILI, V., SELBY, R. and PHILLIPS, T.: 'Metric analysis and data validation across Fortran projects', IEEE Transactions on Software Engineering, 1983, Vol.9, (6), pp.652-663.

24. IYENGAR, S., PARAMESWARAN, N. and FULLER, $\mathrm{J} .:$ 'A measure of logical complexity of programs', Computer Languages, 1982, Vol.7, pp.147-160.

25. SANSOM, W., NEVILL, D. and DOGARD, P.: 'Predictive software metrics based on a formal specification', Information and Software Technology, 1987, Vol.29, (5), pp.242-248.

26. CURTIS, B., SHEPPARD, S., MILLIMAN, P., BORST, M. and LOVE, T.: 'Measuring the psychological complexity of software maintenance tasks with Halstead and McCabe metrics', IEEE Transactions on Software Engineering, 1979, Vol.5, (2), pp.96-104.

27. PRATHER, R.: 'An axiomatic theory of software complexity measure', The Computer Journal, 1984, Vol.27, (4), pp.340-347.

28. DAVIS, J. and LeBLANC, R.: 'A study of the applicability of complexity measures', IEEE Transactions on Software Engineering, 1988, Vol.14, (9), pp.1366-1372.

29. SHEPPERD, M.: 'A critique of cyclomatic complexity as a software metric', Software Engineering Journal, 1988, Vol.3, (2), pp.30-36.

30. BOEHM, B.: 'Software Engineering Economics', (Prentice-Hall, 1981).

31. MOORES, T.: 'A model to size the development of Prolog programs', Department of Information Systems, City Polytechnic of Hong Kong, Hong Kong, Working Paper No.13, April 1994.

32. McCABE, T. and BUTLER, C.: 'Design complexity measurement and testing', Communications of the ACM, 1989, Vol.32, (12), pp.1415-1425. 